\title{
Target similarity effects: Support for the parallel distributed processing assumptions
}

\author{
MICHAEL S. HUMPHREYS \\ University of Queensland, Brisbane, Queensland, Australia \\ GERALD TEHAN \\ University of Southern Queensland, Toowoomba, Queensland, Australia \\ and \\ ANNISSA O'SHEA and SCOTT W. BOLLAND \\ University of Queensland, Brisbane, Queensland, Australia
}

\begin{abstract}
Recent research has begun to provide support for the assumptions that memories are stored as a composite and are accessed in parallel (Tehan \& Humphreys, 1998). New predictions derived from these assumptions and from the Chappell and Humphreys (1994) implementation of these assumptions were tested. In three experiments, subjects studied relatively short lists of words. Some of the lists contained two similar targets (thief and theft) or two dissimilar targets (thief and steal) associated with the same cue (ROBBERY). As predicted, target similarity affected performance in cued recall but not free association. Contrary to predictions, two spaced presentations of a target did not improve performance in free association. Two additional experiments confirmed and extended this finding. Several alternative explanations for the target similarity effect, which incorporate assumptions about separate representations and sequential search, are rejected. The importance of the finding that, in at least one implicit memory paradigm, repetition does not improve performance is also discussed.
\end{abstract}

The most common representational assumption in contemporary memory research is that memories are stored separately. This assumption can be incorporated into a formal model (e.g, Flexser \& Tulving, 1978; Shiffrin \& Steyvers, 1997), or it can be expressed via such expressions as "finding a memory" or "searching for a memory." A frequent accompaniment to the assumption that memories are stored separately is the assumption that memories are accessed via some form of sequential search. In contrast, our recent work has started with the assumption that memories are stored as a composite and that memory access necessarily involves a parallel process.

Our research has not only started from a different point, it has actively sought to provide support for that starting point. In doing this, we have been guided by formal models incorporating composite representations and parallel access (Chappell \& Humphreys, 1994; Humphreys, Bain, \& Pike, 1989). Although the models clearly accommodate the predictions we test, they do not require those predictions. That is, a failure to confirm the prediction would not necessarily invalidate the basic assumptions of the

This research was supported by Australian Research Council Grant A79531860 to the first two authors. The authors thank Richard Shiffrin, Glen Smith, and Doug Nelson for their helpful comments on earlier drafts. Correspondence should be addressed to M. S. Humphreys, Department of Psychology, University of Queensland, Brisbane, QLD 4072, Australia (e-mail: mh@psy.uq.edu.au). model, because we might have been wrong about some of the auxiliary assumptions about the nature of the materials we are using, the instructions we use, or the procedures we employ. Nevertheless, a persistent failure to confirm the prediction while using a variety of materials and procedures would be evidence against the basic assumptions.

It is also the case that we cannot support our basic assumptions by disproving the alternative assumptions (separate storage and sequential search). Indeed, we suspect that it is probably impossible, using behavioral data alone, to distinguish between separate storage and sequential search on the one hand and composite storage and parallel access on the other. Nevertheless, we think that our approach is valuable because we ask different questions from those we would have asked if we had started with a different set of basic assumptions. We are also frequently able to disprove specific versions of theories that incorporate assumptions about separate storage and sequential search or provide data that, while not disproving a theory, restricts future developments of that theory.

The interaction between targets at the time of retrieval is one example of a phenomenon that is easily explained via assumptions about composite memories and parallel access but is not as readily explained by assumptions about separate storage and sequential search. In fact, the later class of models may attempt to explain target interaction by assuming that the interaction is the result of storage processes (Shiffrin \& Steyvers, 1997) or the result of mediation (Barnes \& Underwood, 1959). 
In our investigations of target interactions, we have conducted a large number of experiments examining proactive interference (PI) in a short-term cued recall paradigm (Tehan \& Humphreys, 1995, 1996, 1998). In these experiments, the subjects studied two four-item blocks of words before receiving a single extralist cue for one of the items in Block 2. The cue either was presented immediately after the last item in Block 2 or was delayed for 2 sec. PI was manipulated by having another item related to the cue present in Block 1 . Thus, the cue might be ANIMAL, the target cat in Block 2, and the interfering item dog in Block 1. Under these conditions, we consistently observed PI on a delayed test but not on an immediate test. However, when we used an ending cue (e.g., the cue is ENCH, the Block 2 target bench, and the Block 1 interfering item wrench), we consistently observed PI on both immediate and delayed tests.

In order to explain the differential effect of test delay on PI with semantic and ending cues, we proposed that a short-lasting phonemic code protected the target from interference on an immediate test. The assumption here was that the phonemic code would be effective in discriminating cat from dog but not bench from wrench. On the basis of our assumptions that memories were stored as a composite and that the list words were simultaneously activated by a list or context cue, we then asked the question as to whether we could present the semantic and phonemic information in different words (Tehan \& Humphreys, 1998). To test this hypothesis, we presented a rhyme $(\log )$ of the interfering item $(\log )$ in Block 2 and were able to show that this increased PI. We then took these ideas one step further by presenting the phonemes of the interfering item in three different Block 2 words (e.g., the interfering item is dog, and the Block 2 words are dart, mop, and fig plus the target cat). This manipulation also increased PI.

Collectively, the Tehan and Humphreys (1998) experiments provide relatively direct evidence for composite memories and for the simultaneous activation of the list items. Note in particular that the items used to enhance PI are unrelated to the cue, so they are extremely unlikely to be activated by the cue. This suggests that something like a contextual or list cue is activating all of the list items. These findings are also relatively difficult to explain using ideas about separate storage and sequential search. In this paper, we address these same two assumptions about simultaneous activation and composite storage. Although we are primarily interested in testing the basic assumptions of simultaneous activation and composite storage, it is useful to refer to a concrete example of a model that incorporates these ideas. This example serves as an existence proof that the ideas in question can lead to the predicted results.

Our existence proof relies on the connectionist model proposed by Chappell and Humphreys (1994). In the Chappell and Humphreys model, associations between items or between an item and itself are stored in the weights that connect one set of features with the other set. In this model, episodic information is stored as context- to-item associations, semantic information as item-toitem associations, and lexical information as intraitem associations. In applying this model to cued recall with an extralist cue, we start with the observation that, in this paradigm, the cue was not paired with the target during study. ${ }^{1}$ This aspect of the paradigm requires the model to combine information derived from the cue and information derived from the target in order to produce an item that is associated with the cue and was in the list (Humphreys, Wiles, \& Bain, 1993; Humphreys, Wiles, \& Dennis, 1994; Wiles, Humphreys, Bain, \& Dennis, 1991). In order to produce the desired result, the context cue is assumed to activate all of the items in the study list and suppress all other items. In addition, the extralist-associate cue is assumed to activate all of its associates and suppress all other items. Because the model uses distributed representations, the elements that will remain active are the elements that are activated by the cue and the context. Thus, if a word is an associate of the cue and in the list, its elements will remain active. That is, the model is calculating an approximation to the intersection between the associates of the cue and the items in the list. Elements also remain active whenever the representation of a nontarget item in the list partially overlaps the representation of one of the associates of the cue. These partial overlaps provide a major source of noise in the model.

The presence of noise in the output of the intersection process necessitates a cleanup or deblurring process. To model this process, Chappell and Humphreys (1994) employed an autoassociative lexical memory. That is, prior to learning a list of words, a set of potential items (patterns) was stored in the autoassociative memory. For the present purposes, the relevant property of an autoassociative memory is that it can complete an incomplete input or clean up a noisy input. That is, if only some of the elements in a prelearned pattern are turned on, the autoassociator can complete the pattern by turning on the remaining elements. Similarly, if the entire prelearned pattern is presented plus noise, the autoassociator can turn off the noise, converging to the prelearned pattern.

In this paper, we are primarily concerned with response competition (two or more items are subsumed under the same cue). Because the autoassociator is highly nonlinear, it is difficult to say just what will happen, in a particular case, when two patterns plus noise remain in the intersection. However, mathematical analysis and simulations show that one of three events can occur. The autoassociator can converge to one of the two patterns. In this case, we would say that the pattern that remains active corresponds to the recalled item. Another possibility is that the autoassociator converges to a null state where no elements are active. The final possibility is that the autoassociator converges to a state where every element is active. Both of the latter two possibilities seem to correspond to a state where response competition has prevented the recall of either response. That is, the two targets have blocked or mutually inhibited each other. Furthermore, this blocking of both alternatives is more likely to occur when the two patterns have few overlaps. Thus, the Chap- 
pell and Humphreys (1994) model suggests that it should be possible to change the degree or nature of the interactions between targets by varying target overlap, which we will operationalize as target similarity.

The Chappell and Humphreys (1994) model produces priming in free association by strengthening the intraitem associations. This means that the autoassociator is more likely to converge to a primed item than to an unprimed item. However, because the model does not use a context cue in free association, all associates of the cue will compete to determine which one is produced, not just those that are in the study list. Thus, in free association, the amount of competition between two associates will be essentially the same when both are in the study list and when only one is in the study list. However, the priming assumption that was used in the model does predict that response repetition will have similar effects on free association and cued recall.

\section{EXPERIMENTS 1A, 1B, AND 1C}

Experiments 1A, 1B, and 1C employed high- and lowtarget-similarity conditions. In the high-similarity condition, subjects studied a list containing two targets, which were morphemic relatives (e.g., thief and theft) and were associated with a common cue (ROBBERY). In the low-similarity condition, the two list targets (e.g., thief and steal) were relatively unrelated associates of the same common cue (ROBBERY). The morphemic relatives used would be judged by subjects to be semantically, phonologically, and graphemically similar, and we assume that the memory code could include any or all of these components.

To determine whether there were different target interaction effects in the high- and low-similarity conditions, we had planned to compare the probabilities of recalling at least one of the two targets in the high-similarity condition with the same probability in the low-similarity condition. Our expectation was that this probability would be higher in the high-similarity condition and that this would indicate that, at times, the two dissimilar targets were inhibiting each other or that the similar targets were facilitating each other. Although, in Experiment 1A, there was a clear difference in the predicted direction, there was a measurement problem, which made it difficult to unambiguously interpret this difference. That is, the base rates (the probability of producing the competitors when they were studied by themselves) were not equal. We tried to rectify this problem in Experiment $1 \mathrm{~B}$ by using a slightly different set of materials.

Because the base rates were still not equal in Experiment $1 \mathrm{~B}$, we decided to compare the observed probability of producing one of two targets, when both were studied, with the predicted probability of producing one of two targets. This prediction was based on the production of the targets when they were studied by themselves (this procedure will be described in more detail later). In order to collect data that were more appropriate for this new statistic, we conducted Experiment $1 \mathrm{C}$. The only change from
Experiment 1B was in the list positions in which the competitor and target were presented. This comparison between predicted and obtained probabilities had the added advantage of providing an indication of whether there was facilitation (target recall greater than independence) with two similar targets or inhibition (target recall less than independence) with two dissimilar targets.

Half of the subjects in each experiment were given free association instructions (use the cue to produce the first word that comes to mind), and half were given cued recall instructions (use the cue to recall a related word from the list that has just been studied). The use of free association instructions necessitated the use of procedures to disassociate, in the subject's mind, the free association task and the study list. Our procedures to do this were unusual, but the results show that they were highly successful.

Subjects studied relatively short lists of words (the critical lists contained 12 or 13 words) under runningmemory-span instructions. That is, they were told that when the list stopped, they would be required to recall the last four (Experiment 1A) or last three (Experiments 1B and 1C) words in the list. Since the subjects never knew in advance when the list would end, these instructions ensured that they paid attention to all of the list items. It also ensured that they rehearsed in three- or four-item blocks. Since two targets were always separated by four intervening items, these instructions helped to prevent the target and its competitor from being rehearsed together. In addition, they provided the cover story for our use of free association instructions. Subjects were told that, on some trials, the recall of the last few items would be delayed while they responded to a cue. It was explained that running memory span was the primary task and that the cue was being presented in order to make running memory span more difficult. For the subjects given free association instructions, we hoped that the running-memory-span task would provide an effective cover story as to why they were being asked to free associate to a cue. We also hoped that requiring subjects to switch attention as soon as they produced a response to the cue would make them less likely to think about their response and/or notice that it had been a word in the list. We also used running-memory-span performance as an additional dependent measure, along with the latency of responding, in an attempt to determine whether the process or processes involved in cued recall take extra time or resources in comparison with free association.

We also included a target repetition condition in the experiments (the target was presented twice with a small number of intervening items). This condition served as a test of the priming assumption in the Chappell and Humphreys (1994) model, which predicts a repetition effect in both cued recall and free association. It also served as a conceptual control to the repetition of morphemic relatives.

\section{Method}

Subjects. Seventy-two subjects were tested in each of Experiments 1A, 1B, and 1C. The subjects were introductory psychology 
students at the University of Queensland who participated as part of a course requirement. In each experiment, an equal number of subjects was randomly assigned to the free association and cued recall conditions.

Materials and lists. Thirty cue-target pairs were selected from the University of South Florida Norms (Nelson, McEvoy, \& Schreiber, 1994). The targets were weak associates (mean strength = $.145, S D=.086$ ) of the cue terms. In addition, two competitors of the target terms, which were also weak associates of the cue terms, were selected. The similar competitor was morphologically related to the target, whereas the dissimilar competitor had no morphological relationship with the target. The mean strength of the association from the cue to the similar and dissimilar competitors were .102 $(S D=.056)$ and $.091(S D=.058)$, respectively. A complete list of sets of cues, targets, and competitors is provided in the Appendix. A small number of these sets were replaced by new sets for Experiments $1 \mathrm{~B}$ and $1 \mathrm{C}$. The new sets were also chosen from the materials in the University of South Florida Norms (Nelson et al., 1994). These new sets were chosen so that the strength of the cue to similar competitor was somewhat weaker or the strength of the cue to dissimilar competitor was somewhat stronger than it had been in the original sets.

For each subject, 42 lists of words were prepared. There were 12 filler lists, each of which contained $4-8$ words, and 30 experimental lists, each of which contained 12 or 13 words. To construct the experimental lists, 11 filler words were chosen so that they were not obviously related to any of the words in the experimental word sets. For each subject, each experimental list was randomly paired with 1 of the 30 experimental word sets, and that set was then randomly assigned to one of six experimental conditions with the proviso that there were an equal number of lists in each condition. The six types of experimental lists defined the within-subjects conditions. The six types of experimental lists used in Experiments 1A and 1B are illustrated in Figure 1. In the target-alone, similar-competitor-alone, and dissimilar-competitor-alone conditions, the appropriate word from the experimental word set was presented as the 7 th word of a 12-word list. In the repetition condition, the target word from the experimental word set was presented as the 3rd and 8th word of a 13-word list. In the target-plus-similar-competitor and target-plusdissimilar-competitor conditions, the competitor was presented as the 3 rd word and the target was presented as the 8th word in a 13word list. The result was that the target word was always the 6th last word in an experimental list; if there were two presentations of targets within an experimental list, the two presentations were always separated by 4 intervening words. Experiment $1 \mathrm{C}$ differed from Experiments $\mathrm{IA}$ and $\mathrm{IB}$ in that all experimental lists consisted of 13 words. In these lists, the target word always occurred in Position 3 , and, if the target was repeated or if a competitor was presented, it occurred in Position 8.

Procedure. The subjects were instructed that they would be presented with a series of words on the screen one at a time. They were told that the lists would vary in length from 4 to 16 words and that they would never know in advance how long the list would be. They were instructed that, when a prompt appeared on the screen, their task was to recall the last 4 words in the list ( 3 words in Experiments $1 B$ and $1 C$ ) in the order in which they were presented. If the subjects could not recall all of the indicated items, they were to recall as many as possible. If they were unable to recall any, they were instructed to respond by saying "none." The words were presented in lowercase at a $1-\mathrm{sec}$ rate. The prompt remained on the screen for $9 \mathrm{sec}$. The experimenter recorded the subject' answers and then initiated the presentation of the next list.

Free association instructions. The subjects were instructed that, if a word appeared in uppercase letters, they were to respond by saying the first related word that came to them. They were given $2 \mathrm{sec}$ to respond to the cue, at which point the prompt appeared, and they were required to perform the running-memory-span task. A voice key was used to record the latency of the free association response. The experimenter recorded the free association response, the runningmemory-span response, and then initiated the presentation of the next list.

Cued recall instructions. The subjects were instructed that, if a word appeared in uppercase letters, they were to respond by recalling a list word that was semantically related to the cue. If they could not recall a related word, they were told to report this by saying the word "none." All other details were the same as with the free association instructions.

\section{Results and Discussion}

The probability of producing the target, the similar competitor, and the dissimilar competitor is presented in Table 1 for each of the six study conditions for both the free-association and cued recall subjects in each of the three experiments. The probability of producing an item that was presented in the study list is presented in bold face. The other probabilities represent extralist intrusions. In all comparisons, the level of significance was set to $p<.05$.

In order to determine whether priming was occurring in the free association conditions, we compared the probability of producing either the target or the competitor, when both were presented (target-plus-similar-competitor and target-plus-dissimilar-competitor conditions), with the same probabilities when only the competitor was presented (similar-competitor-only and dissimilar-competitoronly conditions). Across the three experiments, the probability of producing either of the two presented items was .36 , and the probability of producing either the presented competitor or the nonpresented target was .31. This modest difference suggests that priming may be weak under these conditions. However, it is possible that the effect of priming is being obscured by the need to compare the probability of producing either of two items. We return to the question of priming in Experiments $2 \mathrm{~A}$ and 2B, in which a cleaner comparison was possible.

There are three important differences between cued recall and free association in the results. First, the production of nonpresented words was much lower for cued recall than for free association. There are 10 comparisons that can be made in each experiment between the probability of producing a nonpresented word in free association and the same probability in cued recall. For each of the three experiments, for all 10 comparisons, the probability was lower for cued recall than for free association. In addition, the average production probability for these nonpresented words across all three experiments was .097 for free association and .017 for cued recall.

The second difference between free association and cued recall was the effect of a second presentation of the target term. There was no sign of any additional priming produced by a second presentation of the target word in free association. In contrast, in cued recall, a second presentation of the target word approximately doubled the probability of producing the target. A one-way analysis of variance (ANOVA) comparing target production in the target-alone and repetition conditions conducted on the free association and cued recall conditions from each experiment produced no significant differences for the 

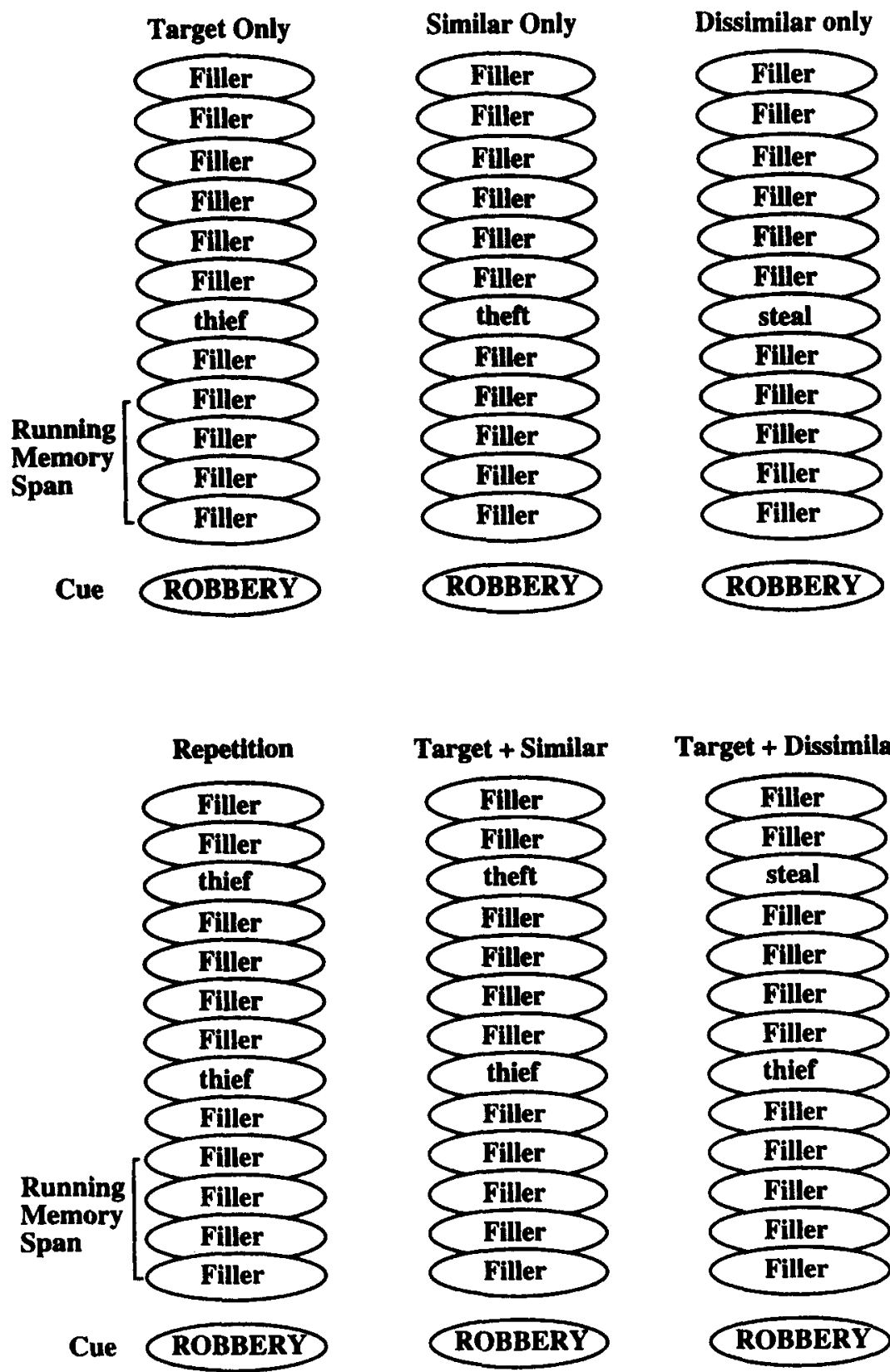

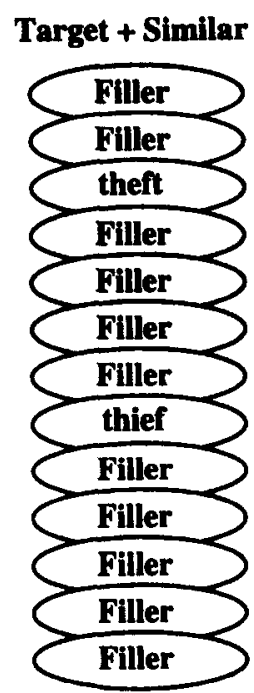

ROBBERY
Target + Dissimilar

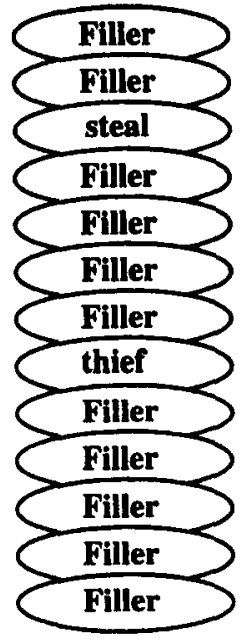

ROBBERY

Figure 1. Examples of the six experimental sequences used in Experiments $1 \mathrm{~A}$ and $1 \mathrm{~B}$. Experiment 1C used similar sequences except that they were always 13 items in length, and a single presentation of the target occurred in Position 3 and the competitors were always presented in Position 8. In the example, the cue is ROBBERY, the target is thief, the similar competitor is theft, and the dissimilar competitor is steal.

free association conditions $\left[F(1,34)=0.33, M S_{\mathrm{e}}=0.04\right.$ $F(1,35)=0.98, M S_{\mathrm{e}}=0.05 ;$ and $F(1,34)=0.22, M S_{\mathrm{e}}=$ 0.04 ; for Experiments 1A, 1B, and 1C, respectively]. All three of the comparisons in the cued recall conditions were significant $\left[F(1,35)=33.83, M S_{\mathrm{e}}=0.03 ; F(1,35)=\right.$ $17.69, M S_{\mathrm{e}}=0.06$; and $F(1,33)=27.51, M S_{\mathrm{e}}=0.04$, for Experiments $1 \mathrm{~A}, 1 \mathrm{~B}$, and $1 \mathrm{C}$, respectively].

The third difference between free association and cued recall relates to the probability of producing at least one of two targets. In the cued recall conditions of all three experiments, the probability of producing at least one of the two targets was greater when two similar targets were studied than when two dissimilar targets were studied, and this effect was not present in free association. This difference in the probability of producing at least one of the two targets does not look like it could have resulted from the relatively small variations in the probabilities of producing the competitors when they were studied by 
Table 1

Probabilities of Producing the Target, the Similar (Sim) Competitor, and the Dissimilar (Diss) Competitor as a Function of Study Condition and Test Instructions in Experiments $1 \mathrm{~A}, 1 \mathrm{~B}$, and $1 \mathrm{C}$

\begin{tabular}{|c|c|c|c|c|c|c|}
\hline \multirow[b]{3}{*}{ Study Condition } & \multicolumn{3}{|c|}{ Free Association } & \multicolumn{3}{|c|}{ Cued Recall } \\
\hline & \multirow[b]{2}{*}{ Target } & \multicolumn{2}{|c|}{ Competitor } & \multirow[b]{2}{*}{ Target } & \multicolumn{2}{|c|}{ Competitor } \\
\hline & & Sim & Diss & & Sim & Diss \\
\hline \multicolumn{7}{|c|}{ Experiment $1 \mathrm{~A}$} \\
\hline Target only & .22 & .14 & .05 & .24 & .03 & .00 \\
\hline Target repetition & .19 & .13 & .07 & .47 & .02 & .01 \\
\hline Target plus & & & & & & \\
\hline $\begin{array}{l}\text { similar competitor } \\
\text { Target plus }\end{array}$ & .19 & .18 & .06 & .31 & .12 & .01 \\
\hline dissimilar competitor & .23 & .13 & .10 & .23 & .05 & .03 \\
\hline $\begin{array}{l}\text { Similar competitor } \\
\text { only }\end{array}$ & .17 & .20 & .05 & .03 & .25 & .01 \\
\hline $\begin{array}{l}\text { Dissimilar competitor } \\
\text { only }\end{array}$ & .14 & .09 & .11 & .00 & .00 & .22 \\
\hline \multicolumn{7}{|c|}{ Experiment $1 \mathrm{~B}$} \\
\hline Target only & .21 & .12 & .06 & .21 & .03 & .01 \\
\hline $\begin{array}{l}\text { Target repetition } \\
\text { Target plus }\end{array}$ & .16 & .17 & .06 & .44 & .03 & .01 \\
\hline similar competitor & .22 & .19 & .07 & .36 & .14 & .01 \\
\hline Target plus & & & & & & \\
\hline dissimilar competitor & .21 & .08 & .12 & .21 & .03 & .11 \\
\hline $\begin{array}{l}\text { Similar competitor } \\
\text { only }\end{array}$ & .13 & .17 & .05 & .04 & .29 & .00 \\
\hline $\begin{array}{l}\text { Dissimilar competitor } \\
\text { only }\end{array}$ & .09 & .10 & .11 & .01 & .01 & .26 \\
\hline \multicolumn{7}{|c|}{ Experiment $1 \mathrm{C}$} \\
\hline Target only & .28 & .10 & .05 & .16 & .02 & .01 \\
\hline $\begin{array}{l}\text { Target repetition } \\
\text { Target plus }\end{array}$ & .31 & .12 & .01 & .42 & .04 & .00 \\
\hline $\begin{array}{l}\text { similar competitor } \\
\text { Target plus }\end{array}$ & .17 & .22 & .06 & .21 & .19 & .00 \\
\hline $\begin{array}{l}\text { dissimilar competitor } \\
\text { Similar competitor }\end{array}$ & .11 & .12 & .22 & .15 & .04 & .15 \\
\hline only & .18 & .23 & .05 & .05 & .31 & .00 \\
\hline $\begin{array}{l}\text { Dissimilar competitor } \\
\text { only }\end{array}$ & .18 & .09 & .15 & .01 & .01 & .26 \\
\hline
\end{tabular}

Note-The probabilities of producing words that were present in the study list are given in bold. Other probabilities represent extralist intrusions.

themselves. However, in order to remove any possible effects of base rate differences in the comparison, we compared the observed probability of producing one of two presented targets with the predicted probability. To calculate the predicted probability for each subject, we took the probability of producing the competitor when it was presented by itself and the probability of producing the target when it was presented by itself. These two probabilities were added together, and then the product of the two probabilities was subtracted from the sum. This is the predicted probability of producing one of two independent events. The observed and predicted probabilities of producing one of the two targets are shown in Table 2.

In free association, there was no consistent relationship between the observed and predicted probabilities, and most of the differences were small. There were also only small differences between the observed and predicted probabilities for the target-plus-similar-competitor conditions in cued recall. In contrast, the observed proba- bility was consistently and substantially smaller than the predicted probabilities for the target-plus-dissimilarcompetitor conditions in cued recall. A one-way ANOVA was used to compare the observed and predicted probabilities for the target-plus-dissimilar-competitor and targetplus-similar-competitor conditions for cued recall and free association in each of the three experiments. The only significant values obtained were two of the three comparisons between observed and predicted probabilities in the target-plus-dissimilar-competitor condition in cued recall $\left[F(1,35)=9.95, M S_{\mathrm{e}}=0.03 ; F(1,33)=5.32, M S_{\mathrm{e}}=\right.$ 0.03 ; for Experiments $1 A$ and $1 C$, respectively]. In addition, the same comparison in Experiment $1 \mathrm{~B}$ approached the adopted level of significance $\left[F(1,35)=3.80, M S_{\mathrm{e}}=\right.$ $0.03, p=.06]$.

We can now add target interaction effects to the previous list of differences between cued recall and free association. In free association, the probability of producing one of two targets was predictable from the assumption of independence and the observed probabilities of producing the targets when they were presented by themselves. This conclusion was not affected by the similarity of the two targets.

In contrast to these free association results, in cued recall, the probability of producing one of two dissimilar targets was consistently less than would be expected given independence. Furthermore, this conclusion was affected by target similarity, since similar targets were produced at the level predicted by the independence assumption.

Turning to the question of whether the process or processes involved in cued recall take extra time or resources in comparison with free association, we examined both response latencies and running-memory-span performance. Because of the relatively low probabilities of producing a target or competitor and the small number of observations per condition, we could not devise a procedure to eliminate outliers among the latencies. Even when we ignored the within-subjects conditions and calculated the median latency for each subject, it was apparent that these

Table 2

Observed and Predicted Probabilities of Producing Either the Target or the Competitor in Cued Recall and Free Association for Target-Plus-Similar-Competitor (Tar-Sim) and

Target-Plus-Dissimilar-Competitor (Tar-Diss) Conditions in Experiments 1A, 1B, and $1 \mathrm{C}$

\begin{tabular}{|c|c|c|c|c|}
\hline & \multicolumn{2}{|c|}{ Cued Recall } & \multicolumn{2}{|c|}{ Free Association } \\
\hline & Tar-Sim & Tar-Diss & Tar-Sim & Tar-Diss \\
\hline \multicolumn{5}{|c|}{ Experiment $1 \mathrm{~A}$} \\
\hline Observed & .42 & $.26 *$ & .37 & .33 \\
\hline Predicted & .41 & .40 & .38 & .31 \\
\hline \multicolumn{5}{|c|}{ Experiment $1 \mathrm{~B}$} \\
\hline Observed & .51 & .32 & .41 & .32 \\
\hline Predicted & .44 & .41 & .35 & .30 \\
\hline \multicolumn{5}{|c|}{ Experiment $1 \mathrm{C}$} \\
\hline Observed & .39 & $.30^{*}$ & .39 & .34 \\
\hline Predicted & .41 & .39 & .46 & .40 \\
\hline
\end{tabular}

*A significant difference between the observed and predicted probabilities. 
medians were spuriously long for some subjects. As an alternative way to compare latencies, we plotted the latencies for all correct responses (responses of items that were present in the study list) separately for the cued recall and free association subjects in Experiments 1A, 1B, and $1 \mathrm{C}$. We then normalized the resulting frequency histograms by dividing the frequency of occurrence in each 100-msec segment by the total number of observations in that condition. These normalized histograms are presented in Figure 2. By inspection, it can be seen that the leading edges were highly similar. In fact, the only real difference between the two distributions appears to lie in the longer right-hand tail of the cued recall distribution. Our conclusion is that, although the average time to respond
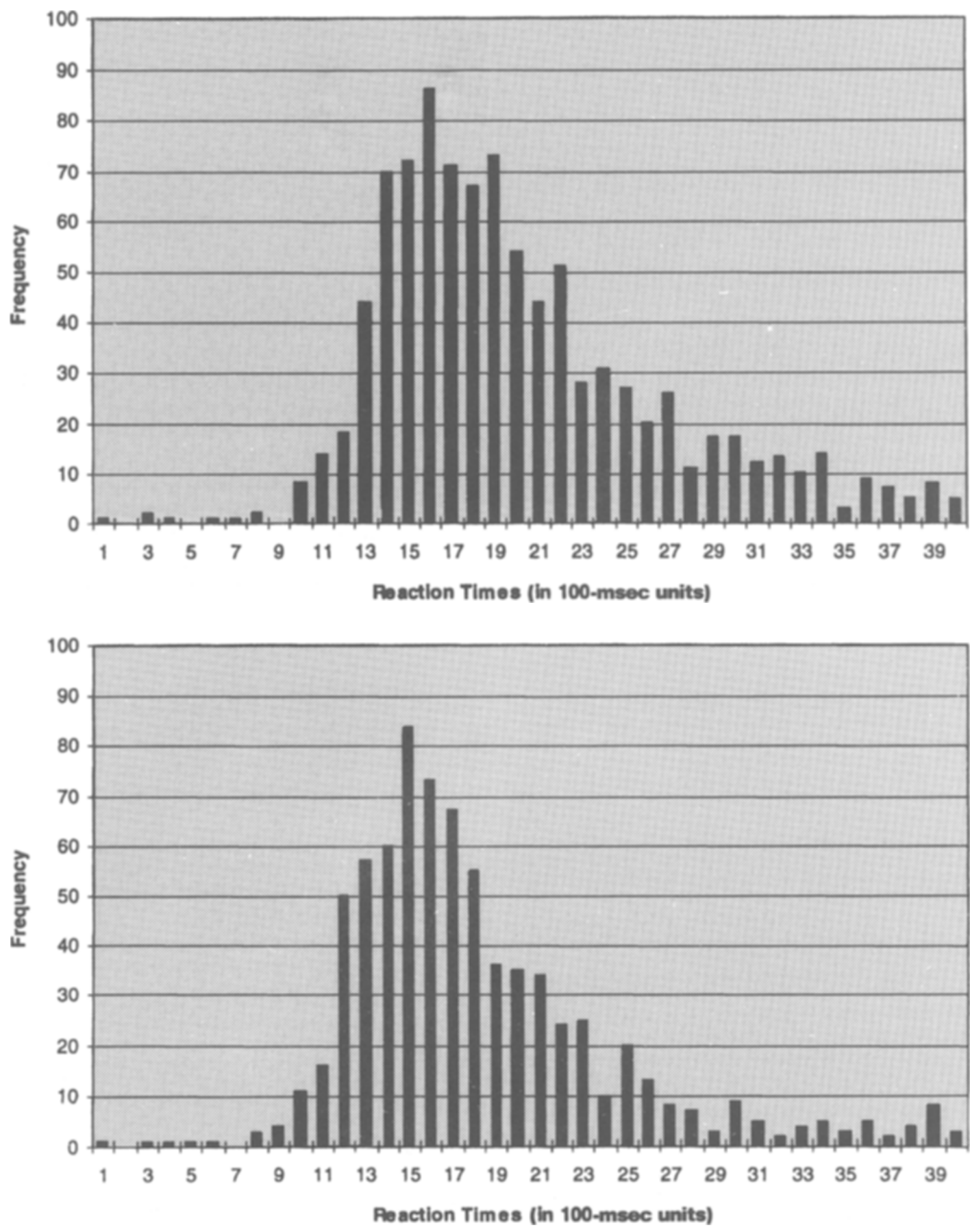

Figure 2. Normalized frequency distributions for reaction time (in 100-msec units) to recall list items in free association and cued recall in Experiments 1A, 1B, and 1C. 
was longer in cued recall, the minimum time to respond was very similar in cued recall and in free association.

We also calculated the mean running-memory-span performance for the control lists (running memory span was tested immediately after the last list word was presented) and for the experimental lists (running memory span was tested after the subject responded to the cue or after $2 \mathrm{sec}$ ). An item was considered correct regardless of the position in which it was recalled. For each experiment and for both the free association condition and the cued recall condition, the average running-memory-span performance on the control and experimental lists is presented in Table 3.

As expected, for the control lists, running-memoryspan performance was not systematically related to whether the subjects were assigned to the free association or cued recall conditions. In contrast, with the experimental lists, where running memory span followed the free association or cued recall response, running-memoryspan performance was always better following cued recall than following free association. Because it seemed possible that the effect of instructions on running memory span were due to the way in which the subjects studied the list items and not to the interfering effect of making a response, for each experiment we tested whether the difference between free association and cued recall instructions was greater for the experimental lists than for the control lists. The results for Experiments $1 \mathrm{~A}$ and $1 \mathrm{~B}$ were significant $\left[F(1,70)=9.25, M S_{\mathrm{e}}=0.10\right.$, and $F(1,70)=6.45$, $M S_{\mathrm{e}}=0.05$, respectively]. The effect was in the same direction for Experiment $1 \mathrm{C}$ but failed to reach significance $\left[F(1,70)=2.87, M S_{\mathrm{e}}=0.06\right]$.

The results of Experiments 1A, 1B, and 1C support two of the predictions that are derived from ideas about composite representations and simultaneous access as embodied in the Chappell and Humphreys (1994) modelnamely, that there are interactions between target terms when two targets are subsumed under the same cue, and these interactions are confined to cued recall. However, they do not support a third prediction that is specific to this model that the effects of repetition would be the same in cued recall and free association. In addition, they produced a substantial amount of data that can be used to

Table 3

Number of Words Correctly Recalled (Ignoring Order) on the Running-Memory-Span Test for the Filler and Experimental Lists in the Cued Recall (CR) and Free Association (FA) Conditions in Experiments 1A, 1B, and 1C

List

\begin{tabular}{cccccccc} 
& \multicolumn{9}{c}{ List } \\
\cline { 2 - 4 } \cline { 5 - 8 } Experiment & CR & FA & Difference & & CR & FA & Difference \\
\hline 1A & 2.89 & 2.84 & .05 & & 1.59 & 1.22 & $.37^{*}$ \\
1B & 2.55 & 2.43 & .12 & & 1.42 & 1.11 & $.31^{*}$ \\
IC & 2.35 & 2.40 & -.05 & & 1.24 & 1.16 & .08 \\
\hline
\end{tabular}

Note-The subjects were asked to recall the last four words in Experiment $1 \mathrm{~A}$ and the last three words in the remaining experiments. *The difference in running-memory-span performance between the cued recall and free association conditions is significantly greater for the experimental lists than for the control lists. constrain theories of cued recall and free association. That is, we know that (1) the probability of recalling at least one of two dissimilar targets is less than would be predicted on the basis of independence, (2) attempting to recall to a cue produces less interference with the recall of the last three or four words from the study list than does free associating to the same cue, (3) the leading edges of the latency distributions are similar for cued recall and free association, and (4) the benefit due to a second presentation at study is found in cued recall but not free association.

Before discussing the implications of these findings for some other memory theories, we wanted to clear up two points raised by these experiments. The first concerned the relatively weak priming effect that was observed. Without strong evidence for a priming effect, it is difficult to argue that our procedure of embedding a free association response within a running-memory-span procedure is producing comparable results to more traditional indirect or implicit memory tests. The second point concerned the lack of a second-presentation benefit in free association. Challis and Sidhu (1993) reported that massed multipresentation benefits were weak but probably present with what they termed conceptual implicit tasks, such as free association. Several investigators have also found spaced repetition benefits with perceptual implicit tasks (Greene, 1990; Logan, 1990; Roediger \& Challis, 1992). However, other investigators have reported null or inconsistent effects (Jacoby \& Dallas, 1981; Kirsner \& Speelman, 1996; Perruchett, 1989). Because of these inconsistencies in the literature, and because we had manipulated instructions between subjects, we felt that it was necessary to replicate our lack of a second-repetition benefit. We were particularly concerned with using conditions in which the failure to find an effect could not be attributed to any differential effect of instructions (free association vs. cued recall) on how the list was studied.

\section{EXPERIMENTS 2A AND 2B}

The issue of whether the instructional manipulation (cued recall vs. free association) was affecting retrieval or storage in the first set of experiments arises because instructions were manipulated between subjects. Thus, it was possible that the expectation for a particular type of retrieval task was affecting how the lists were studied. A within-subjects manipulation of retrieval instructions, in which the subjects did not know what type of retrieval instruction they would receive until after the list had been presented, would have been the ideal solution to this problem. However, pilot work indicated that subjects had a great deal of difficulty when they were simultaneously given the cue and instructions as to how to use it. Instead, we decided to employ a final recognition test in order to show that the failure to find a benefit from a second presentation in free association was due to the retrieval process employed, not to how the list words had been studied.

In Experiments 2A and 2B, only a free association condition was run using the running-memory-span procedure employed in the previous experiments. There were 
three within-list conditions, no target was presented, a single target was presented once, and a single target was presented twice. In addition to determining whether there was a second-presentation benefit, this design enabled us to determine in a direct fashion whether there was a priming effect. After a series of free association and filler trials, subjects were given a final recognition test for some of the items in the lists. The same three within-list conditions were employed in the final recognition test as had been employed in the free association test.

\section{Method}

Subjects. Thirty subjects were tested in Experiment 2A, and 34 were tested in Experiment 2B. The subjects were introductory psychology students at the University of Queensland who participated as part of a course requirement.

Materials. Sixty cue-target pairs were selected from the University of South Florida Norms (Nelson et al., 1994). The association values were in the range of $10 \%-20 \%$. The running-memoryspan study lists consisted of 42 lists. There were 12 control lists, which contained 4-8 words. The subjects were immediately tested for the recall of the last 3 words from the control lists. Illustrations of the experimental lists used in Experiment $2 \mathrm{~A}$ are provided in Figure 3. To create the experimental lists in Experiment 2A, 30 sets of 11 filler words were created. For each subject, 2 of the cue-target pairs were randomly assigned to each of the sets of filler words. For 10 sets of filler words, the 2 assigned targets were included along with 9 of the filler words to form the study list. In these lists, one target was repeated in Positions 3 and 7, and the other was repeated in Positions 4 and 8 (list length $=13$ ). For another 10 sets of filler words, the 2 assigned targets were also included along with all 11 of the fillers to form the study list. One target was presented once in Position 7. and the other was presented in Position 8 (list length = 13). For the remaining 10 sets of filler words, the assigned targets were not included in the study list (list length $=11$ ). For each experimental list, a cue was presented immediately after the last word in the list. If the list contained 2 targets (either both repeated or both presented once), the cue was associated with 1 of the 2 targets. If the list contained no targets, the cue was associated with 1 of the 2 targets that had nominally been assigned to that list. Whether or not it was present at study, a target that was associated with the cue is referred to as a tested target. The recognition test consisted of the nontested targets ( 10 repeated, 10 presented once, and 10 not presented). In addition, the 10 tested targets that were not present in the study list were also included in the recognition test; however, they are not included in any analysis. The presentation of the test words was randomized for each subject.

In Experiment 2B, all experimental lists contained 13 words. Again, each list had 2 assigned targets. For 20 lists, both of the assigned targets were included. One of these targets was repeated ( $\mathrm{Po}-$ sitions 3 and 7 , or Positions 4 and 8 ), and 1 was presented once (Position 8 if the repeated target occurred in Positions 3 and 7, and Position 7 if the repeated target occurred in Positions 4 and 8). For the other 10 lists, the assigned targcts were not included in the study list; so 3 extra fillers were required. The recognition test consisted of 30 nontested targets ( 10 repeated, 10 presented once, and 10 not presented).

Procedure. The free association and running-memory-span procedures were the same as in Experiment $1 \mathrm{~A}, 1 \mathrm{~B}$, and 1C. After the last list had been presented in the running-memory-span/free association section of the experiment, the subjects were given recognition instructions. They were told that they were going to be given a
No Presentation

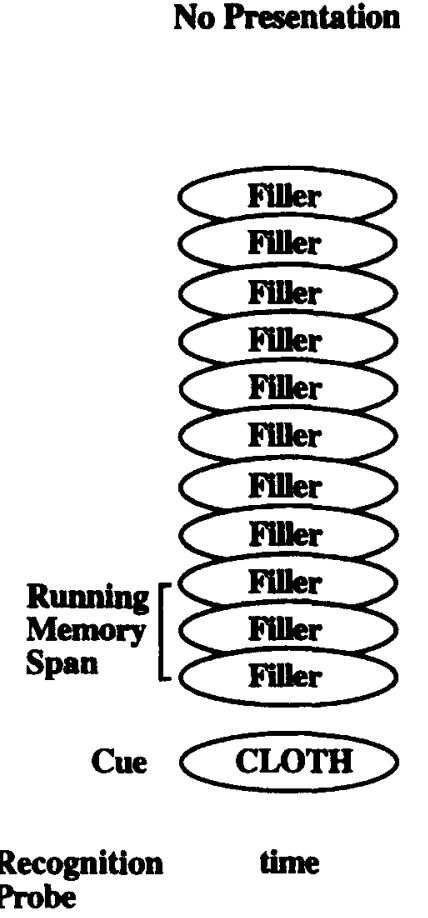

Single Presentation

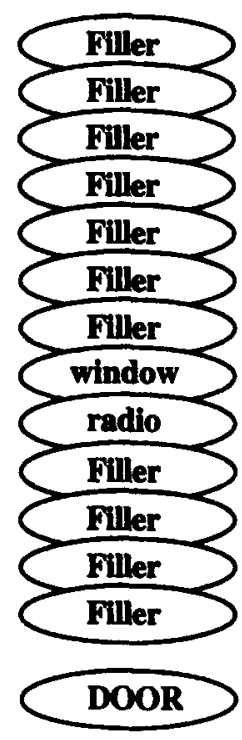

radio
Two Presentations

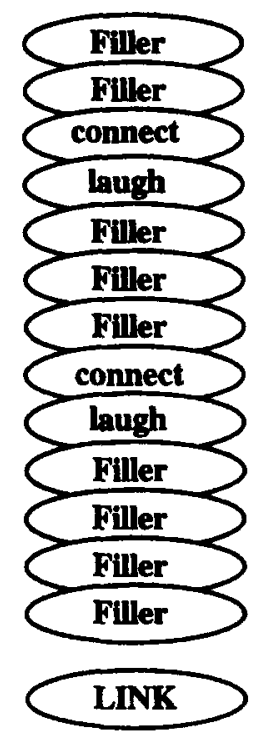

laugh

Figure 3. Examples of the three experimental sequences used in Experiment 2A. Experiment 2B differed in that the single-presentation and two-presentation conditions occurred within the same sequence. In both experiments, the target that was presented first was equally often tested for free association and recognition. The CUE-target pairs used in the examples are CLOTH-material, HOUR-time, STEREO-radio, LINK-connect, and COMEDY-laugh. 
list of words, that they had seen some of these words during the running-memory-span section of the experiment, and that they had not seen some of the words. They were instructed that, for each word, they were to press one of two buttons to indicate whether the word was previously presented or not.

\section{Results and Discussion}

The probability of producing the target word on the free association task is presented in Table 4 for Experiments $2 \mathrm{~A}$ and $2 \mathrm{~B}$. To establish whether there was a priming effect, we compared the average of the single- and two-presentation conditions with the no-presentation condition. This comparison was significant for Experiments $2 \mathrm{~A}$ and $2 \mathrm{~B}\left[F(1,29)=16.18, M S_{\mathrm{e}}=0.02\right.$, and $F(1,33)=16.37, M S_{\mathrm{e}}=0.02$, respectively]. We then compared the single- and two-presentation conditions to determine whether there was a second-presentation benefit. This comparison did not reach significance $[F(1,30)=$ $0.63, M S_{\mathrm{e}}=0.02$; and $F(1,33)=.66, M S_{\mathrm{e}}=0.02$; for Experiments $2 \mathrm{~A}$ and $2 \mathrm{~B}$, respectively].

The probability of recognizing a target word on the final recognition test is presented in Table 5 for Experiments $2 \mathrm{~A}$ and $2 \mathrm{~B}$. To establish whether the subjects could discriminate between old and new words, we compared the average of the repeated and once-presented conditions with the not-presented condition. This comparison was significant for Experiments $2 \mathrm{~A}$ and $2 \mathrm{~B}[F(1,29)=$ $34.29, M S_{\mathrm{e}}=0.02$, and $F(1,33)=37.34, M S_{\mathrm{e}}=0.03$, respectively]. We then compared the repeated and oncepresented conditions to determine whether there was a second-presentation benefit. This comparison was significant for Experiments 2A and 2B $\left[F(1,29)=4.67, M S_{\mathrm{e}}=\right.$ 0.04 , and $F(1,33)=12.11, M S_{\mathrm{e}}=0.02$, respectively].

The results from Experiments $2 \mathrm{~A}$ and $2 \mathrm{~B}$ were very clear. On the immediate free association test, there was a priming effect but no second-presentation benefit. On the final recognition test, old words were discriminated from new words, and there was a second-presentation

Table 4

Probability of Producing the Target on the Free Association Task as a Function of the Repetition Status of the Target in Experiments $2 A$ and $2 B$

\begin{tabular}{lll}
\hline & \multicolumn{2}{c}{ Experiment } \\
\cline { 2 - 3 } Repetition Status & $2 \mathrm{~A}$ & $2 \mathrm{~B}$ \\
\hline No presentation & .14 & .17 \\
Single presentation & .27 & .27 \\
Two presentations & .24 & .29 \\
\hline
\end{tabular}

Table 5

Probability of Identifying a Word as Old on the Final Recognition Test as a Function of the Repetition Status of the Target for Experiments 2A and 2B

\begin{tabular}{lcc} 
Repetition Status of the Target for Experiments 2A and 2B \\
\cline { 2 - 3 } Repetition Status & \multicolumn{2}{c}{ Experiment } \\
\hline No presentation & .25 & 2B \\
Single presentation & .40 & .33 \\
Two presentations & .51 & .49 \\
\hline
\end{tabular}

benefit. Our conclusion is that the lack of a secondpresentation benefit on the immediate free association test in these two experiments and in the previous three experiments was a product of the retrieval process employed and was not a product of the nature of the study process. In addition, it seems highly unlikely that the lack of a second-presentation benefit in free association was due to the subjects' rejecting words, which they recognized as having been in the list. The subject reports indicate that the running-memory-span task was very demanding. In fact, several subjects spontaneously reported that the only way they could perform the running-memory-span task after interference was to complete the interfering task (free association or cued recall) as fast as they could. Under these conditions, the subjects should not have been checking their answers on the free association task.

\section{GENERAL DISCUSSION}

We found target similarity effects in cued recall, but not in free association. These results, along with Tehan and Humphreys's (1998) results, provide support for the assumptions that (1) memories are stored as composite representations and (2) the items in the study are simultaneously activated at the time of retrieval. ${ }^{2}$ Note that, in this study, the argument for simultaneous activation is not as straightforward as it was in Tehan and Humphreys's (1998) study. That is, Tehan and Humphreys showed that items that were unrelated to the cue were still involved in the retrieval process. Because they were unrelated to the cue, they must have been activated by something other than the cue. In the present study, the two interacting items were both related to the cue. However, the interaction was only present in free association, not in cued recall. The use of a context or list cue in cued recall, but not in free association, provides a parsimonious explanation for the pattern of results obtained across the two studies.

In addition the pattern of results (performance with dissimilar targets was less than predicted by independence), our dependent measure (the probability of recalling either target) and the auxiliary results about reaction time and running-memory-span performance allow us to reject a variety of alternative cued recall theories. We also found that the second presentation of a target in a list produced no discernible effect on free association, though it had a large effect on cued recall and recognition. This finding was unexpected. It allows us to reject one of the specific assumptions in Chappell and Humphreys's (1994) model. It may also indicate a more general problem with the way priming has been conceptualized. This issue will be discussed after we examine the alternative cued recall theories.

\section{Alternative Theories \\ Mediation. Barnes and Underwood (1959) suggested that mediation was a better explanation than response generalization for the large amount of positive transfer found in the $A B-A B^{\prime}$ paradigm. That is, they suggested that when trying to recall $B^{\prime}$, subjects would first recall}


$B$ and then use $B$ to recall $B^{\prime}$. Mediation can explain an enhanced recall of $B^{\prime}$, but it cannot explain an enhanced recall of either $B$ or $B^{\prime}$, since one of them must be recalled in order for it to mediate the recall of the other.

Storage processes. Target interactions could also result from storage processes. We consider three different possibilities here. First, subjects could rehearse the first target when the second target is presented. In our situation, this was unlikely because the two targets were separated by four words, and the running-memory-span instructions would have caused the subjects to rehearse in blocks of four (Experiment 1A) or three (Experiments $1 \mathrm{~B}$ and $1 \mathrm{C}$ ). An extra rehearsal of the 1 st presented target should also have produced a primacy effect. However, in Experiment $1 \mathrm{C}$, in which the target was presented before the competitor, target recall and competitor recall were equal. In contrast, in Experiments $1 \mathrm{~A}$ and $1 \mathrm{~B}$, in which the target was presented after the competitor, target recall was considerably better than competitor recall. If anything, this is evidence for a slight recency effect, not a primacy effect.

Interference effects at storage have also been suggested as an explanation for PI in the Brown-Peterson paradigm (Gorfein, 1987). If this were occurring, it should also produce a primacy effect. In addition, Tehan and Humphreys (1996) tested this hypothesis by presenting two targets such as hydrogen and zinc, and then they either provided a cue that subsumed both targets (CHEMICAL ELEMENT) or a cue that only subsumed the second target (METAL). There was substantial interference when the cue subsumed both targets and no interference when the cue only subsumed the second target. This experiment was repeated using rhyming words and cuing with either the rhyme, which subsumed both words, or a stem, which subsumed only one of the words. Again, PI was found only when the cue subsumed both targets.

Finally, Shiffrin and Stevyers (1997) incorporated the following storage assumption in REM:

When a word presented for study calls to mind a previous image, and when that image matches the presented word features to a high enough degree, then all storage occurs in the recovered image, and a new image is not stored. (p. 155)

They also went on to assume that learning would be impaired when a word is stored in a previous image. In combination, these two assumptions imply that the first target in the list should be recalled better than the second target. ${ }^{3}$ As we have noted previously, this does not occur for either the target-plus-similar-competitor condition or the target-plus-dissimilar-competitor condition. In addition, the Tehan and Humphreys (1996) procedure, in which a cue uniquely specifies one of the two targets in a list, is a direct test of the hypothesis that similar words can be stored in the same image, reducing performance on the second word. However, this test would be more powerful if the two targets were presented in the same block or list of items and if morphemic relatives were used in place of the less similar category exemplars. Finally, it seems possible for REM to predict that the probability of recalling either of two similar targets is better than the probability of recalling either of two dissimilar targets. However, it does not seem possible for REM to predict that the probability of recalling either of two dissimilar targets is less than would be expected assuming independence. This occurs because REM, like its predecessor SAM, has no provision for two images to compete with each other so that neither image is retrieved.

Generate-Recognize. The traditional generaterecognize explanation for cued recall with an extralist cue assumes that the semantic cue would be used by itself to generate a string of potential targets from within the semantic memory system (Humphreys et al., 1989; Jacoby \& Hollingshead, 1990). ${ }^{4}$ After generating a target, it is assumed that the subject attempts to recognize that target using the episodic system. In such a model, there are many ways that having a second similar item in the list would increase the probability of recognition.

However, when taken as a whole, the evidence from the present experiments strongly suggests that a generaterecognize process is unlikely. The first point to note is that a generate-recognize process does provide an explanation for the low level of extralist intrusions in cued recall. However, the Chappell and Humphreys (1994) model and the other ideas put forward by Wiles et al. (1991) about how to compute an "intersection" also predict that there will be a low level of extralist intrusions. In fact, it is this low level of extralist intrusions that caused Humphreys et al. (1993) and Wiles et al. (1991) to characterize the necessary computation as the computation of an "intersection."

Response latencies do provide some support for a generate-recognize process. That is, average latencies are clearly longer in cued recall than in free association, which is consistent with the assumption that cued recall requires an additional process (recognition). However, the leading edges of the latency distributions are very similar, and this is unlikely to occur if almost all of the generated items are subject to a recognition check. Furthermore, in order for a generate-recognize model to produce the doubling of the probability of cued recall with a second study presentation and the low level of extralist intrusions, most of the items generated would have to be subject to a recognition check. However, there are too many unknowns (e.g., the speed of recognition and the probability of producing the target on a second or third generation attempt) to construct a rigorous argument. Nevertheless, it seems possible that a study expressly designed to examine cued recall and free association latencies under highly speeded conditions could establish that the fastest cued recall responses are also accurate. This would indicate that a recognition check was not needed in order to largely restrict responses to the list.

The running-memory-span results, for which free association was more disruptive than cued recall, are, however, clearly incompatible with the traditional generaterecognize process. That is, generating an alternative using 
semantic memory, then performing a recognition check using episodic memory, and then either producing the generated alternative or generating another alternative should not be less disruptive than a process simply employing the generation and production stages.

In contrast the finding that there is greater interference with running-memory-span performance after free association than after cued recall can be accommodated within the Chappell and Humphreys (1994) framework. That is, in this framework, we would assume that context is involved in retrieving a list item using an extralist cue and in retrieving the last three or four words from a list. That is, after the free association response, the subject would have to reinstate the list context in order to perform the running-memory-span task. In contrast, because the same contextual cue would be used for both cued recall and running memory span, there would be no additional need to reinstate it. For an additional discussion of the role of contextual reinstatement in memory, see Dennis and Humphreys (1998).

The final evidence against a generate-recognize process comes from a comparison of the observed and predicted probabilities of recalling at least one of the two dissimilar targets presented in a list. In cued recall, the observed probability was consistently less than the predicted probability, but this did not occur in free association or with similar targets in cued recall. This finding suggests that two dissimilar targets block each other, such that neither one is produced. This kind of blocking is inconsistent with a generate-recognize process in which one target blocks another only if it is generated and recognized.

\section{Repetition Effects in Free Association and Other Implicit Memory Tasks}

In Experiments 1 A, 1B, and 1C, the second presentation of a target did not increase free association, even though it had a large effect on cued recall. In Experiments $2 \mathrm{~A}$ and $2 \mathrm{~B}$, the second presentation also had no effect on free association, although it had a large effect on a subsequent recognition test. This is a null result, but we have obtained it in five different experiments. In addition, each of these experiments had a substantial sample size and produced a strong result using an explicit memory task.

Although our finding that there is no benefit to free association from a second presentation seems reliable, the generality of this finding cannot be ascertained at this time. Challis and Sidhu (1993) consistently found a null effect when they used massed presentations and a perceptual implicit task (also see Weldon, Roediger, Beitel, \& Johnston, 1995). In contrast, Challis and Sidhu reported a small effect of massed repetitions on a conceptual implicit task. Our finding of a null effect using free association (a conceptual implicit task) casts doubt on Challis and Sidhu's generalization that the important contrast is between perceptual and conceptual implicit tasks.

There are also several instances in the literature where spaced repetitions have produced benefits in perceptual implicit tasks (e.g., Greene, 1990; Jacoby \& Dallas, 1981;
Perruchett, 1989; Roediger \& Challis, 1992). However, in all of these studies, there were also failures to find effects of repetition, and, when effects were found, they were typically small. This inconsistent and confusing pattern of results may be due to any one of a number of task variables. However, it is also suggestive of an involvement of explicit memory processes in at least some of these studies. In this regard, we think that our procedures are very effective in reducing the possibility that subjects in our free association conditions will deliberately attempt to recall a word from the study list.

Finally, Logan's (1990) procedures are representative of tasks in which multirepetition benefits are typically found. ${ }^{5}$ In these tasks, subjects perform the same task on every trial (e.g., lexical decision), whereas, in the literature that has just been reviewed, subjects perform a different task at study and test (e.g., read at study and complete stems at test).

The issue about when repetition does not improve implicit memory performance is potentially critical for our understanding of implicit memory. If we substitute producing for identifying, the basic idea behind the priming mechanism used by Chappell and Humphreys (1994) has been expressed in verbal form by Uttl and Graf (1996):

The degree of unitization (or the self coordinating nature) of a target's representation is a positive function of practicing the skills required for identifying that target and priming is viewed as reflecting an increase in skill (at identifying that target) that was brought about by recently encountering the target. (p. 88)

It is also consistent with the more general ideas that priming is a form of skill learning (Logan, 1990) and with the assumption that priming depends on a procedural memory system (Squire, 1992). However, a memory system that asymptotes after a single presentation would not look like any known example of skill learning or procedural memory. There may well be elements of skill learning in many priming paradigms (e.g., this may be likely when subjects perform the same task at study and test, or it may be more likely with perceptual implicit tasks). However, finding even a single paradigm in which there is a large effect on explicit tests due to a second presentation but no effect on implicit tests is a theoretical challenge.

\section{REFERENCES}

Barnes, J. M., \& Underwood, B. J. (1959). "Fate" of first-list associations in transfer theory. Journal of Experimental Psychology, 58, 97-105.

Challis, B. H., \& SidHu, R. (1993). Dissociative effect of massed repetition on implicit and explicit memory tasks. Journal of Experimental Psychology: Learning, Memory, \& Cognition, 19, 115-127.

Chappell, M., \& Humphreys, M. S. (1994). An auto-associative neural network for sparse representations: Analysis and application to models of recognition and cued recall. Psychological Review, 101, 103-128.

Dennis, S., \& Humphreys, M. S. (1998). The role of context in episodic recognition: The bind cue decide model of episodic memory. Unpublished manuscript, University of Queensland.

Dyne, A. M., Humphreys, M. S., Bain, J. D., \& Pike, R. (1990). As- 
sociative interference effects in recognition and recall. Journal of Experimental Psychology: Learning, Memory, \& Cognition, 16, 813-824.

FleXser, A. L., \& TulviNG, E. (1978). Retrieval independence in recognition and recall. Psychological Review, 85, 153-171.

GorFEIN, D. S. (1987). Explaining context effects on short-term memory. In D. S. Gorfein \& R. R. Hoffman (Eds.), Memory and learning. The Ebbinghaus Centennial Conference (pp. 153-172), Hillsdale, NJ: Erlbaum.

GraF, P., \& Schacter, D. L. (1987). Selective effects of interference on implicit and explicit memory for new associations. Journal of $E x$ perimental Psychology: Learning, Memory, \& Cognition, 13, 45-53.

GREENE, R. L. (1990). Spacing effects on implicit memory tasks. Journal of Experimental Psychology: Learning, Memory, \& Cognition, 16, 1004-1011

Humphreys, M. S., Bain, J. D., \& Pike, R. (1989). Different ways to cue a coherent memory system: A theory for episodic, semantic and procedural tasks. Psychological Review, 96, 208-233.

Humphreys, M. S., Wiles, J., \& BaIN, J. D. (1993). Memory retrieval with two cues: Think of intersecting sets. In D. E. Meyer \& S. Kornblum (Eds.), Attention and performance XIV: Synergies in experimental psychology, artificial intelligence, and cognitive neuropsychology-A silver jubilee (pp. 489-508). Cambridge, MA: MT Press.

Humphreys, M. S., Wiles, J., \& DeNNIS, S. (1994). Toward a theory of human memory: Data structures and access processes. Behavioral \& Brain Sciences, 17, 655-667.

JACOBY, L. L., \& DALlAS, M. (1981). On the relationship between autobiographical memory and perceptual learning. Journal of Experimental Psychology: General, 110, 306-340.

JACOBY, L. L., \& HolLINGSHEAD, A. (1990). Toward a generate/recognize model of performance on direct and indirect tests of memory. Journal of Memory \& Language, 29, 433-454.

KIRSNER, K., \& SPEELMAN, C. (1996). Skill acquisition and repetition priming: One principle, many processes? Journal of Experimental Psychology: Learning, Memory, \& Cognition, 22, 563-575.

LOGAN, G. D. (1990). Repetition priming and automaticity: Common underlying mechanisms? Cognitive Psychology, 22, 1-35.

Nelson, D. L., McEvoy, C. L., \& Schreiber, T. (1994). The University of South Florida Word Association, Rhyme and Word Fragment Norms. Unpublished manuscript, University of South Florida, Tampa.

OSTERGAARD, A. L. (1998). The effects on priming of word frequency, number of repetitions, and delay depend on the magnitude of priming. Memory \& Cognition, 26, 40-60.

Perruchett, P. (1989). The effect of spaced-practice on explicit and implicit memory. British Journal of Psychology, 41, 320-331.

RaAiJMAKers, J. G., \& ShIFFrin, R. M. (1981). Search of associative memory. Psychological Review, 88, 93-134.

Roediger, H. L., III, \& Challis, B. H. (1992). Effects of exact repetition and conceptual repetition on free recall and primed word fragment completion. Journal of Experimental Psychology: Learning, Memory, \& Cognition, 18, 3-14.

Shiffrin, R. M., \& STEYVERS, M. (1997). A model for recognition memory: REM-Retrieving effectively from memory. Psychonomic Bulletin \& Review, 4, 145-166.

SQuIRE, L. R. (1992). Memory and the hippocampus: A synthesis from findings with rats, monkeys, and humans. Psychological Review, 99, 195-231.

Tehan, G., \& Humphreys, M. S. (1995). Transient phonemic codes and immunity to proactive interference. Memory \& Cognition, 23, 181-191.

Tehan, G., \& HumPHREYs, M. S. (1996). Cuing effects in short-term recall. Memory \& Cognition, 24, 719-732.

Tehan, G., \& Humphreys, M. S. (1998). Creating proactive interference in immediate recall: Building a dog from a dart, a mop, and a fig. Memory \& Cognition, 26, 477-489.

Tulving, E., \& Thomson, D. M. (1973). Encoding specificity and retrieval processes in episodic memory. Psychological Review, 80, 352-373.

UTTL, B., \& GRAF, P. (1996). Object orientation information in semantic and episodic memory. Canadian Journal of Experimental Psychology, 50, 87-103.

WELDON, M. S., \& COLSTON, H. L. (1995). Dissociating the generation stage in implicit and explicit memory tests: Incidental production can differ from strategic access. Psychonomic Bulletin \& Review, 2 381-386.

Weldon, M. S., Roediger, R. L., III, BeItel, D. A., \& JohnSton, T. R. (1995). Perceptual and conceptual processes in implicit and explicit tests with picture fragment and word fragment cues. Journal of Memory \& Language, 34, 268-285.

Wiles, J., Humphreys, M. S., Bain, J. D., \& Dennis, S. (1991). Direct memory access using two cues: Finding the intersection of sets in a connectionist model. In R. P. Lippman, J. E. Moody, \& D. S. Touretzky (Eds.), Advances in neural information processing systems 3 (pp. 635641). San Mateo, CA: Morgan Kaufmann.

\section{NOTES}

1. The assumption that the extralist cue can access the memory for the list target because it was, in some sense, stored with the target (e.g., it was implicitly activated by the target at study or because the encoding of the target overlapped the encoding of the cue) has a long history (Raaijmakers \& Shiffrin, 1981; Tulving \& Thomson, 1973). The difficulties this hypothesis has in accounting for specific aspects of cued recall performance have been reviewed elsewhere (Humphreys et al., 1989; Humphreys, Wiles, \& Dennis, 1994). Even more importantly, in Tehan and Humphreys's (1998) experiments, words that are unrelated to a cue can influence which of two targets is retrieved to that cue. Factors other than encoding factors must be invoked in order to explain this finding.

2. An anonymous reviewer has suggested that our results can be subsumed by Graf and Schacter's (1987) generalization that associative interference occurs only in explicit memory tasks. However, this generalization is now known to be at least partially incorrect. Dyne, Humphreys, Bain, and Pike (1990) showed that when recall was eliminated from pair recognition (an explicit task), there was no associative interference. In addition, the present results show that both free association and cued recall are affected by competition (the presence of an alternative target in the study list) but that the nature of the competition varies. In contrast, Graf and Schacter had subjects study two pairs in which the same cue was paired with different targets and then tested them by providing the cue plus the unique stem of one of the words paired with that cue. Under these conditions, there was no associative interference with implicit memory instructions, but there was with explicit memory instructions. In our experiments, it is possible to equate performance at a below independence level with interference and performance at the level predicted by independence with the lack of interference. Thus, it is possible to tell a very general story about the relationship between the Graf and Schacter results and our results. However, this attempt to fit the procrustean bed of the explicit/implicit distinction to associative interference is oversimplified. A more complete story will require a specification of how the information derived from the cue and the stem is combined and some ideas about the processes that allow these two sources of information to work together to eliminate interference. The combining of information from two sources has been extensively discussed by Chappell and Humphreys (1994), Humphreys et al. (1993), Humphreys et al. (1994), and Wiles et al. (1991), and it is a relatively straightforward matter to apply these ideas to the cue-plus-stem task. The creation of such a model, however, goes beyond the concerns of the present paper.

3. When REM has been applied to recall, it has been assumed that the cue and the target were studied together. While an application to cued recall with an extralist associate is likely to be similar in many ways, such an application is not straightforward within the REM or the earlier SAM framework (Humphreys et al., 1993), and it is possible that some of these predictions will change.

4. Weldon and Colston (1995) tested what they referred to as the generate-recognize explanation for the differences between explicit and implicit memory tasks. In their test, they included a cue-plus-stem condition, in which subjects studied weak associates (e.g., ocean wave) and were then given the first member of the pair plus the stem of the target as a cue (e.g., ocean wa). On the basis of their results, they concluded "that the generation process during intentional retrieval differs from that during incidental retrieval" (p. 385). We agree that their results support this conclusion with respect to the cue-plus-stem task, but we can see no justification for an extension to tasks involving extralist cues. That is, there is a joint occurrence of the cue and the target in the study list in 
the cue-plus-stem task, but not when recall is cued with an extralist cue (see note 1).

5. Even when the study and test trials are the same, multirepetition benefits effects are still somewhat variable. Kirsner and Speelman (1996) did not find such a benefit. One difference between their study and Logan's (1990) lies in the density of the repetitions. Logan had 16 repetitions within $3 \mathrm{~min}$ and Kirsner and Speelman had 1 repetition per day. Ostergaard (1998) has suggested that, under these conditions, priming, in general, is somewhat variable and may depend on a variety of task conditions, such as baseline performance. What is clear is that, at this time, we do not have an adequate understanding of the conditions that produce repetition benefits.

APPENDIX

The Cues, Their Associated Targets, and the Similar and Dissimilar Competitors Used in Experiment 1

\begin{tabular}{|c|c|c|c|}
\hline \multirow[b]{2}{*}{ Cue } & \multirow[b]{2}{*}{ Target } & \multicolumn{2}{|c|}{ Competitor } \\
\hline & & Similar & Dissimilar \\
\hline HATE & like & dislike & enemy \\
\hline TENSE & tight & uptight & loose \\
\hline PERSON & human & man & friend \\
\hline SELECT & choose & choice & few \\
\hline INMATE & prisoner & prison & convict \\
\hline WORSHIP & praise & pray & church \\
\hline BRAIN & think & thought & mind \\
\hline POLLUTION & dirty & dirt & waste \\
\hline COUNSEL & advise & advice & people \\
\hline LAWN & mower & mow & green \\
\hline CONTEST & winner & win & game \\
\hline SEPARATE & apart & part & split \\
\hline ANNOUNCE & speak & speaker & yell \\
\hline CHORUS & singer & song & line \\
\hline DOUBLE & twin & twice & single \\
\hline TAPE & record & recorder & cassette \\
\hline FRAGILE & break & breakable & delicate \\
\hline INHALE & breath & breathe & smoke \\
\hline VACUUM & cleaner & clean & suck \\
\hline ALCOHOL & drink & drunk & drug \\
\hline TEMPER & angry & anger & tantrum \\
\hline CRISP & crunchy & crunch & fresh \\
\hline UNCONSCIOUS & asleep & sleep & coma \\
\hline ROBBERY & thief & theft & steal \\
\hline COURAGE & strength & strong & bravery \\
\hline AMUSE & funny & fun & happy \\
\hline EMPLOYEE & worker & work & boss \\
\hline EDUCATION & teacher & teach & learn \\
\hline VARY & differ & different & change \\
\hline PROTECTION & safety & safe & guard \\
\hline
\end{tabular}

(Manuscript received September 8, 1998;

revision accepted for publication July 13, 1999.) 